\title{
ON HOMOGENEOUS HEREDITARILY UNICOHERENT CONTINUA
}

\begin{abstract}
G. R. GORDH, JR. ${ }^{1}$
ABSTRACT. Let $\pi$ denote the class of all hereditarily unicoherent Hausdorff continua in which each indecomposable subcontinuum is irreducible. It is shown that if the continuum $M$ in $\pi$ is decomposable, then the set of weak terminal points of $M$ is a nonempty, proper subset. The following generalization of a theorem of $\mathrm{F}$. Burton Jones is an immediate corollary: if the continuum $M$ in $\pi$ is homogeneous, then $M$ is indecomposable. As an application, it is proved that if $X$ is a homogeneous, hereditarily unicoherent Hausdorff continuum which is an image of an ordered compactum, then $X$ is an indecomposable metrizable continuum.
\end{abstract}

1. Introduction. F. Burton Jọnes has proved that every homogeneous hereditarily unicoherent metric continuum is indecomposable [7]. It is the primary purpose of this note to give a short, simple proof of Jones' theorem. At the same time, we extend his theorem to the class $\mathbb{M}$ of all hereditarily unicoherent Hausdorff continua in which each indecomposable subcontinuum is irreducible. Our proof is based on the observation that if $M$ is a decomposable continuum in $M$, then the set of weak terminal points of $M$ is a nonempty, proper subset (hence $M$ cannot be homogeneous).

As an application of our result in the nonmetric setting, we prove that if $X$ is a homogeneous hereditarily unicoherent Hausdorff continuum which is an image of an ordered compactum, then $X$ is an indecomposable metrizable continuum.

2. Definitions and preliminary remarks. By a continuum we mean a compact, connected Hausdorff space. A continuum is hereditarily unicoherent if the intersection of any two of its subcontinua is connected.

Received by the editors June 6,1974 .

AMS (MOS) subject classifications (1970). Primary 54F 15, 54F 20; Secondary 54F05, 54E35.

Key words and phrases. Hausdorff continuum, hereditarily unicoherent, homogeneous, indecomposable, image of ordered compactum, metrizability.

1 During this research the author was visiting the University of Zagreb on an exchange program sponsored jointly by the National Academy of Sciences (U.S.A.) and the Yugoslav Academy of Sciences and Arts. 
Notation. Throughout this paper $M$ will denote the class of all continua $M$ such that

(i) $M$ is hereditarily unicoherent, and

(ii) every indecomposable subcontinuum of $M$ is irreducible.

Remark. The class $M$ was defined and studied in [4]. It is well known that every hereditarily unicoherent metric continuum belongs to M (e.g., [6]). The class $M$ may coincide with the class of all hereditarily unicoherent continua; i.e., condition (ii) may be superfluous. In fact, it has been conjectured that every indecomposable continuum is irreducible. Some partial results concerning this problem are contained in [1], [3], and [5]. We shall use the fact that continua in $M$ contain maximal irreducible subcontinua $[2$, Theorem 3.7].

Let $X$ be a hereditarily unicoherent continuum. If $x$ and $y$ are points of $X$, then $\langle x, y\rangle$ will denote the unique subcontinuum of $X$ which is irreducible from $x$ to $y$. A point $p$ of $X$ is said to be a terminal point of $X$ if each irreducible subcontinuum containing $p$ is of the form $\langle p, x\rangle$ for some $x$ in $X[10]$. We define a point $p$ of $X$ to be a weak terminal point if there exists a point $x$ in $X$ such that $\langle p, x\rangle$ is a maximal irreducible subcontinuum of $X$.

If $M$ belongs to $M$, then (using the fact that every point is contained in a maximal irreducible subcontinuum) it follows immediately that every terminal point of $M$ is a weak terminal point. The converse is false. For example, the dyadic solenoid is a homogeneous indecomposable continuum in $M$ which consists entirely of weak terminal points, but which contains no terminal point.

The reader is referred to [6] for definitions of undefined terms and for a general discussion about continua.

3. Homogeneity and weak terminal points.

Lemma 1 . If the continuum $M$ is decomposable and belongs to $M$, then the set of weak terminal points of $M$ is a nonempty, proper subset.

Proof. According to Theorem 3.7 of [2], M contains a maximal irreducible subcontinuum. Thus the set of weak terminal points of $M$ is nonempty. Let $M=A \cup B$ where $A$ and $B$ are proper subcontinua. Choose $a \in A-B$, $b \in B-A$, and $p \in\langle a, b\rangle \cap(A \cap B)$. Suppose that $p$ is a weak terminal point of $M$ and let $\langle p, x\rangle$ be a maximal irreducible subcontinuum. Assume that $x$ $\epsilon B$. Since $p \in\langle a, b\rangle \subset\langle a, x\rangle \cup\langle x, b)$, either $p \in\langle a, x\rangle$ or $p \in\langle x, b\rangle$. If $p$ $\epsilon\langle a, x\rangle$, then $\langle p, x\rangle \subset\langle a, x\rangle-\{a\}$ since $\langle p, x\rangle \subset B$. This contradicts the assumption that $\langle p, x\rangle$ is maximal. Thus we may assume that $p \in\langle x, b\rangle$ and 
$p \notin\langle a, x\rangle$. Observe that $b \notin\langle a, p\rangle \subset A$. Also $b \notin\langle a, x\rangle$; for otherwise $p \epsilon$ $\langle a, b\rangle \subset\langle a, x\rangle$ contrary to our assumption. Thus $\langle p, x\rangle \subset\langle a, p\rangle \cup\langle a, x\rangle \subset M$ - $\{b\}$. In particular, $\langle p, x\rangle \subset\langle x, b\rangle-\{b\}$, which contradicts the assumption that $\langle p, x\rangle$ is maximal. It follows that $p$ is not a weak terminal point, and the proof is complete.

As an immediate consequence of Lemma 1 we obtain our generalization of Jones' theorem.

Theorem 1. If $M$ is a homogeneous continuum which belongs to $\mathbb{M}$, then $M$ is indecomposable.

Proof. If $M$ is decomposable, then (by Lemma 1 ) there exist points $p$ and $q$ in $M$ such that $p$ is a weak terminal point and $q$ is not. This contradicts the assumption that $M$ is homogeneous.

We also obtain a characterization of the indecomposable continua in $M$.

Theorem 2. Let $M$ be a continuum in $M$. Then $M$ is indecomposable if and only if each point of $M$ is a weak terminal point.

Proof. If each point of $M$ is a weak terminal point, then $M$ is indecomposable by Lemma 1 . If $M$ is indecomposable, then $M$ is irreducible. Consequently $M$ contains two disjoint composants, say $C_{1}$ and $C_{2}$ (see [6]). If $p \notin C_{i}(i=1,2)$, then $M=\langle p, x\rangle$ for each $x \in C_{i}$. It follows that each point of $M$ is a weak terminal point.

4. Homogeneous images of ordered compacta. A continuum $X$ is said to be an image of an ordered compactum if there exists a compact totally ordered space $K$ and a continuous surjection $f: K \rightarrow X$. A survey of the most important facts concerning such spaces is given in [9].

Lemma 2. If $X$ is an indecomposable continuum which is an image of an ordered compactum, then $X$ is metrizable.

Proof. If $X$ is not metrizable, then some pair of points $\{p, q\}$ separates $X$ [12]. Let $X-\{p, q\}=U \cup V$ be a separation. Then $X-U$ has at most two components, so $X$ contains a proper subcontinuum with nonempty interior. According to Theorem 3-41 of [6], $X$ is decomposable.

Lemma 3. If $X$ is a hereditarily unicoherent continuum which is an im. age of an ordered compactum, then $X$ belongs to $M$.

Proof. If $X$ is an image of an ordered compactum, then so is every subcontinuum of $X$. By Lemm a 2, every indecomposable subcontinuum of $X$ is 
metrizable, hence irreducible. Consequently $X$ belongs to $\mathbb{M}$.

Theorem 3. If $X$ is a homogeneous, hereditarily unicoherent continuum which is an image of an ordered compactum, then $X$ is an indecomposable metrizable continuum.

Proof. By Lemma 3, $X$ belongs to M. According to Theorem $1, X$ is indecomposable. Thus, by Lemma $2, X$ is metrizable.

Remark. Neither conclusion in Theorem 3 is valid without the assumption that $X$ is hereditarily unicoherent; i.e., there exist decomposable nonmetrizable homogeneous continua which are images of ordered compacta. Such an example can be obtained by identifying the endpoints of a nonmetrizable ordered continuum which is homeomorphic to each of its nondegenerate subcontinua (see [11]).

5. Some questions. (1) Is every (hereditarily unicoherent) indecomposable continuum irreducible?

(2) Is every homogeneous, hereditarily unicoherent continuum indecomposable?

According to Theorem 1, an affirmative answer to (1) implies an affirmative answer to (2).

(3) Is every homogeneous continuum which is an image of an ordered compactum either metrizable or a "generalized simple closed curve" (i.e., a continuum obtained by identifying the endpoints of some ordered continuum)?

It was observed in [7] that Theorem 1 implies that every nondegenerate homogeneous plane continuum which does not separate the plane is indecomposable. More recently, F. Burton Jones has proved that such a continuum is actually hereditarily indecomposable [8]. However the following question, raised in [7], is still unanswered.

(4) Is every nondegenerate homogeneous plane continuum which does not separate the plane a pseudo-arc?

\section{REFERENCES}

1. D. P. Bellamy, Composants of Hausdorff indecomposable continua: a mapping approach, Pacific J. Math. 47 (1973), 303-309.

2. G. R. Gordh, Jr., Monotone decompositions of irreducible Hausdorff continua, Pacific J. Math. 36 (1971), 647-658. MR 43 \#6882.

3. Indecomposable Hausdorff continua and mappings of connected linearly ordered spaces, Proc. Univ. of Oklahoma Conf. on General Topology, 1972.

4. - Terminal subcontinua of hereditarily unicoherent continua, Pacific J. Math. 47 (1973), 457-464.

5. Indecomposable Hausdorff continua and mappings of connected linearly ordered spaces, Glasnik Mat. 9 (29) (1974), 137-139. 
6. J. G. Hocking and G. S. Young, Topology, Addi son-Wesley, Reading, Mass., 1961. MR 23 \#A2857.

7. F. B. Jones, Certain homogeneous unicoherent indecomposable continua, Proc. Amer. Math. Soc. 2 (1951), 855-859. MR 13, 573.

8. - Homogeneous plane continua, Proc. Auburn Univ. Conf. on General Topology, 1969, pp. 46-56.

9. S. Mardešić, On the Hahn-Mazurkiewicz problem in nonmetric spaces, Proc. Second Prague Topological Sympos., 1966, pp. 248-255.

10. H. C. Miller, On unicoherent continua, Trans. Amer. Math. Soc. 69 (1950), 179-194. MR 12, 118.

11. L. B. Treybig, Concerning homogeneity in totally ordered, connected topological spaces, Pacific J. Math. 13 (1963), 1417-1421. MR 28 \#2526.

12. Concerning continua which are continuous images of compact ordered spaces, Duke Math. J. 32 (1965), 417-422. MR 32 \#4673.

INSTITUTE OF MATHEMATICS, UNIVERSITY OF ZAGREB, ZAGREB, YUGOSLAVIA

Current address: Department of Mathematics, Guilford College, Greensboro, North Carolina 27410 